\title{
Simvastatin reduces tumor cell adhesion to human peritoneal mesothelial cells by decreased expression of VCAM-1 and $\beta_{1}$ integrin
}

\author{
BRITTA JANINA WAGNER ${ }^{1,2,5}$, STEFAN LÖB ${ }^{1,2}$, DENNIS LINDAU ${ }^{1}$, HELEN HÖRZER ${ }^{1,3}$, \\ BRIGITTE GÜCKEL ${ }^{3}$, GERD KLEIN ${ }^{4}$, JÖRG GLATZLE ${ }^{2}$, HANS-GEORG RAMMENSEE ${ }^{1}$, \\ BJÖRN LUCAS DIETRICH MARIA BRÜCHER ${ }^{2}$ and ALFRED KÖNIGSRAINER ${ }^{2}$
}

\author{
${ }^{1}$ Department of Immunology, University of Tübingen, Auf der Morgenstelle $15 ;{ }^{2}$ Department of General, Visceral, \\ and Transplantation Surgery, Comprehensive Cancer Center, University of Tübingen, Hoppe-Seyler-Str. 3; \\ ${ }^{3}$ Department of Obstetrics and Gynecology, University of Tübingen, Calwer Str. 7, D-72076 Tübingen; \\ ${ }^{4}$ University Medical Clinic, Center for Medical Research, Section for Transplantation Immunology \\ and Immunohematology, University of Tübingen, Waldhörnlestr. 22, D-72072 Tübingen, Germany
}

Received June 6, 2011; Accepted July 11, 2011

DOI: 10.3892/ijo.2011.1167

\begin{abstract}
Peritoneal carcinomatosis describes cancer metastasis onto the surface of the peritoneum. It is frequently caused by ovarian and colorectal cancer. Once a tumor has penetrated the peritoneum, cancer cells disseminate into the abdominal cavity. Additionally, surgery can account for the spread of free tumor cells. Their subsequent adhesion to mesothelial cells (HMCs) initiates peritoneal carcinomatosis. Therefore, this study analyzed the effect of simvastatin on tumor cell adherence. HMCs were isolated from human greater omentum. Fluorescence-labeled tumor cells (SKOV-3, OvCar-29, OAW42, FraWü; ovarian/HT29; colorectal) were incubated on confluent mesothelial monolayers with $10 \mu \mathrm{M}$ simvastatin for $48 \mathrm{~h}$. Adhesion was quantified using a fluorescence reader. Expression of the adhesion molecules VCAM-1, ICAM- 1 and $\beta_{1}$ integrin chain under the influence of simvastatin $0.1-100 \mu \mathrm{M}$ for $24-72 \mathrm{~h}$ was analyzed using flow cytometry. Simvastatin significantly reduced the adhesion of all ovarian cancer cells and HT29 to HMCs $(\mathrm{P} \leq 0.001)$. Concomitantly simvastatin decreased the expression of VCAM-1 on HMCs. ICAM- 1 and $\beta_{1}$ integrin chain expression on ovarian cancer cells was also clearly reduced. By contrast, the expression of the analyzed adhesion molecules on HT29 cells remained unchanged. Simvastatin clearly inhibits tumor cell adhesion to HMCs. In the case of ovarian cancer cell lines it appears to be mediated by decreased expression of both
\end{abstract}

Correspondence to: Dr Britta Janina Wagner, Present address: ${ }^{5}$ Alexianer Krankenhaus Maria-Hilf, Department of Thoracic, Visceral and General Surgery, Diessemer Bruch 81, D-47805 Krefeld Germany

E-mail: bj.wagner@gmx.net

Key words: peritoneal carcinomatosis, adhesion molecules, simvastatin, ovarian cancer cells, colorectal cancer cells
VCAM-1 on HMCs and the integrin $\alpha_{4} \beta_{1}$ on tumor cells. As an example of adhesion molecule down-regulating drugs, simvastatin may provide a novel therapeutic approach to the prevention of peritoneal carcinomatosis.

\section{Introduction}

Peritoneal carcinomatosis is associated with a median survival of less than one year and has long been regarded as incurable (1). In contrast to the majority of solid tumors, metastatic spread in ovarian cancer is primarily into the abdominal cavity (2). Approximately three-quarters of ovarian cancer patients are diagnosed in the advanced stages FIGO III, characterized by peritoneal metastases throughout the abdominal cavity, or IV, defined by distant metastases (3). Only 20-25\% of patients with advanced stages can be cured (4). Even early-stage ovarian cancer is characterized by peritoneal carcinomatosis in the pelvis (IIb) or by ascites positive for tumor cells (IIc), with a recurrence rate of $40-50 \%$ (5).

Despite advances in the early detection of colorectal cancer, peritoneal lavage fluid of colorectal cancer patients obtained prior to resection proved to be positive in 3-28\% (6). About one-third of patients with pT3/4 colorectal cancer die of peritoneal carcinomatosis (7). Curative resection of colon and rectal cancer were followed by peritoneal carcinomatosis in $4-12 \%$ and $2-19 \%$, respectively (6). The median survival of patients with peritoneal carcinomatosis can be significantly increased by cytoreductive surgery followed by hyperthermic intraperitoneal chemotherapy (1); however, up to $80 \%$ of the patients still experience a recurrence in the first year after surgery (8).

Human mesothelial cells (HMCs) cover the peritoneum (9). The degree of surgical trauma to mesothelial surfaces correlates directly with the rate of tumor recurrence $(10,11)$. Further, exfoliated cancer cells during resection of primary tumors correlated with intra-peritoneal tumor recurrence (12-14). Surgery induces a sterile inflammatory response, which results in up-regulation of adhesion molecules such as intercellular cell adhesion molecule 
(ICAM)-1 and vascular cell adhesion molecule (VCAM)-1 on HMCs $(15,16)$. Ovarian cancer cell adhesion to HMCs is dependent on VCAM-1 (17). VCAM-1-blocking reduced tumor cell adhesion and growth in vitro and in an in vivo mouse model (17). Further, $\alpha_{4} \beta_{1}$ integrin, a known ligand of VCAM-1, is expressed by ovarian cancer cells and mediates adhesion to HMCs $(17,18)$. Intra-abdominal injection of heparin reduced ICAM-1 expression on HMCs and prevented peritoneal colorectal tumor growth in an in vivo rat model (19). Additionally, colorectal cancer cell adherence to human umbilical vein endothelial cells (HUVECs) is mainly ICAM-1-dependent (20). In summary, the expression levels of ICAM-1, VCAM- 1 and $\alpha_{4} \beta_{1}$ integrin appear to correlate with the level of tumor cell adhesion to HMCs in vitro and tumor growth in vivo. Down-regulation of these adhesion molecules would therefore be a promising target to reduce abdominal inoculation of cancer cells.

One potential agent for preventing peritoneal adhesion is simvastatin, a 3-hydroxy-3-methylglutaryl coenzyme A (HMG$\mathrm{CoA})$ reductase inhibitor. Simvastatin belongs to the lipophilic statins, achieving higher levels of exposure in non-hepatic tissues, while hydrophilic statins are primary hepatoselective (21). Besides its use as a lipid-reducing drug, further evidence suggests that systemic statin use could extend to prevention and treatment of different cancers $(22,23)$. Statins have been found to reduce the expression of VCAM- 1 and ICAM-1 significantly in HUVECs (24). This is associated with reduced adherence of monocytes and lymphocytes (25). Furthermore, simvastatin prevents the growth and metastasis of squamous cell carcinoma in the head and neck through down-regulation of the $\beta_{1}$ integrin chain (26).

Due to limited therapeutic options and poor prognosis of peritoneal carcinomatosis patients there is a need to prevent peritoneal inoculation. The process of tumor cell adhesion appears to be a potential target for preventive treatment. The present study therefore investigated whether simvastatin reduces tumor cell adhesion to HMCs via VCAM-1, ICAM-1 and/or $\beta_{1}$ integrin chain down-regulation in an in vitro model of peritoneal carcinomatosis.

\section{Materials and methods}

Materials. Medium 199 (M199), type II collagenase and CellTrace CFSE Cell Proliferation Kit were obtained from Invitrogen (Carlsbad, CA). Heparin sodium was supplied by Ratiopharm (Ulm, Germany). Iscove's modified Dulbecco's medium (IMDM) was from Lonza (Basel, Switzerland); human serum (HS) and endothelial cell growth supplement (ECGS) from PromoCell (Heidelberg, Germany). Fibronectin, propidium iodide (PI) and PKH67 Cell Linker Kit were supplied by Sigma-Aldrich (St. Louis, MO). Formaldehyde solution, fetal calf serum (FCS), accutase, penicillin/streptomycin solution and bovine serum albumin (BSA) were purchased from Fluka Chemicals (Buchs, Switzerland), PAA Laboratories (Pasching, Austria) and Carl Roth (Karlsruhe, Germany), respectively. Simvastatin and dimethyl sulfoxide (DMSO) were from Calbiochem/Merck (Darmstadt, Germany). Simvastatin was dissolved in DMSO and stored as stock solutions of $\geq 10^{-2} \mathrm{M}$ at $-20^{\circ} \mathrm{C}$. The final concentrations of DMSO did not exceed $0.01 \%$ (vol/ $/ \mathrm{vol}$ ). The following anti-human antibodies (unless otherwise indicated) were obtained from Abcam (Cambridge,
UK): fluoroscein isothiocyanate (FITC)-conjugated anti-mouse cytokeratin 18 IgG1 (clone DC10), phycoerythrin (PE)-labeled donkey polyclonal to rabbit $\operatorname{IgG}$, rabbit IgG isotype control and rabbit polyclonal to glyceraldehyde 3-phosphate dehydrogenase (GAPDH); for adhesion-blocking experiments, monoclonal anti-ICAM-1 (clone B-H17), anti-VCAM-1 (clone B-K9) and anti- $\alpha_{4}$-integrin (clone HP2/1). Further anti-human VCAM-1 IgG1 (clone STA, PE-labeled), anti-human ICAM-1 IgG2a (clone MEM-11, FITC-labeled) and the isotype controls mouse IgG2a (clone MOPC-173, FITC-labeled) and mouse IgG1 (clones MOPC-21, PE-labeled; MG1-45, purified) were obtained from BioLegend (San Diego, CA). Cytofix/ Perm, Perm/Wash and mouse anti-human $\beta_{1}$-integrin IgG1 (CD29; clone MAR4, PE-labeled) were from BD Biosciences (Heidelberg, Germany). The monoclonal antibodies against human integrin $\alpha_{9}$-chain $\left(\alpha_{9} \beta_{1}\right.$; clone Y9A2) and the integrin $\beta_{1}$-chain (CD29; clone 4B4) were purchased from AbD Serotec (Raleigh, NC) and Beckman Coulter (Brea, CA), respectively. Recombinant human VCAM-1 and tumor necrosis factor (TNF) were from R\&D Systems (Wiesbaden, Germany) and PeproTech (Hamburg, Germany), respectively.

Cell isolation and culture. HMCs were isolated from the greater omentum from consenting patients undergoing elective surgery. This method was approved by the ethics committee of the Faculty of Medicine and University Hospital at the University of Tübingen, Germany. The isolation procedure was based on the method described by Stylianou et al (27). After enzymatic disaggregation, HMCs were grown on fibronectin-coated dishes in nutrition medium containing M199 supplemented with $20 \mathrm{mmol} / \mathrm{l}$ HEPES (pH 7.4), $2 \mathrm{mmol} / 1$ glutamine, $5 \%$ HS, $15 \%$ FCS, ECGS $(150 \mu \mathrm{g} / \mathrm{ml}), 5 \mathrm{IU} / \mathrm{ml}$ heparin, penicillin $(100 \mathrm{IU} / \mathrm{ml})$, and streptomycin $(100 \mu \mathrm{g} / \mathrm{ml})$. HMCs were assessed by uniform cobblestone appearance at confluence, using transmitted-light microscopy. Additionally, they were tested as positive for cytokeratin 18 using flow cytometry (data not shown). For the experiments, cells were incubated with nutrient-deficient medium without growth factor. HMCs from the same isolation of one donor were partly pre-incubated with $10 \mathrm{ng} / \mathrm{ml} \mathrm{TNF}$ for $15 \mathrm{~h}$, mimicking inflammatory conditions, as mentioned in the results. Unstimulated and inflammatory conditions were tested in pairwise fashion with HMCs derived from the same individuals.

SKOV-3, OvCar-29, OAW42 and FraWü tumor cells were derived from ovarian cancer and HT29 cells from colorectal cancer. SKOV-3, OvCar-29 (subclone of OvCar-3) and HT29 were purchased from the American Type Culture Collection (ATCC; HTB-77, HTB 161 and HTB-38, respectively). OAW42 was from the European Collection of Cell Cultures (ECACC; 85073102). The cell line FraWü was derived from a malignant ascites effusion of an ovarian cancer patient and established in our laboratory. FraWü was authenticated by expression of different cancer/testis antigens (i.e., MAGE-1,-3,-4) and overexpression of self antigens (i.e., HER-2/neu) (28). They were cultured in IMDM with $10 \%$ FCS, penicillin $(100 \mathrm{IU} / \mathrm{ml})$, and streptomycin $(100 \mu \mathrm{g} / \mathrm{ml})$. During the experiments, the cells were incubated with nutrient-deficient HMC culture medium, which did not affect cell survival. For all adhesion assays, the vitality of tumor cells was $\geq 95 \%$ after dyeing, which was quantified by trypan-blue staining. 
Flow cytometry analysis. The effect of simvastatin on the expression of VCAM-1, $\beta_{1}$ integrin chain and ICAM-1 on HMCs and tumor cells was assessed using flow cytometry. They were treated with simvastatin for 24-72 $\mathrm{h}$ at concentrations varying from 0.01 to $100 \mu \mathrm{M}$. DMSO and medium served as controls. All cells were removed from the 24-well culture dish with accutase, as this mild treatment hardly affects cell surface molecule expression. For extracellular staining they were incubated with anti-VCAM-1, anti-ICAM- 1 and anti- $\beta_{1}$ integrin chain fluorescence-labeled antibodies or isotype controls at $4^{\circ} \mathrm{C}$ for $20 \mathrm{~min}$. For intracellular staining of cytokeratin 18 and GAPDH, the cells were permeabilized with $100 \mu \mathrm{l}$ of Cytofix/Perm solution for $20 \mathrm{~min}$ at $4^{\circ} \mathrm{C}$. Perm/Wash buffer was used for subsequent staining steps. For primary antibody staining, rabbit polyclonal to GAPDH or rabbit $\mathrm{IgG}$, as isotype control, were incubated for $30 \mathrm{~min}$ at $4^{\circ} \mathrm{C}$. Staining with the secondary antibody, donkey polyclonal to rabbit IgG, was performed similarly. PI staining was used to analyze cell viability during simvastatin treatment. All cell suspensions were examined using the FACSCalibur cytometer (BD Biosciences). The data were analyzed on the basis of mean fluorescence intensity (MFI) using CellQuest Pro software.

Fluorescence reader analysis. HMC monolayers were grown to confluence on 24-well culture dish. As adhesion molecule down-regulation by simvastatin reached its maximum at $10 \mu \mathrm{M}$ after $48 \mathrm{~h}$, adhesion assays were performed under these conditions. Incubation with medium and DMSO served as controls. All blocking experiments were performed at a concentration of $5 \mu \mathrm{g} / \mathrm{ml}$ of blocking antibody. The reduction in tumor cell adhesion to HMCs was at its maximum at this concentration (data not shown). The blocking antibodies were added $30 \mathrm{~min}$ before tumor cells were applied to the HMC monolayers. Incubation was carried out in presence of the antibodies. CFSE staining of tumor cells followed the instructions of the manufacturer (Invitrogen). Cells $\left(10^{6}\right)$ were stained with a final CFSE concentration of $7.5 \mu \mathrm{M}$. Tumor cells $\left(10^{5}\right)$ were added to the confluent HMCs corresponding to a tumor cell-HMC ratio of 1:1. After incubation for $48 \mathrm{~h}$, each well was washed three times with PBS and fixed in $1 \%$ formaldehyde/PBS. The plates were measured directly using a Wallac 1420 Victor Multilabel Counter (Perkin-Elmer, USA). The arbitrary fluorescence units (AFU) corresponded to the number of adherent tumor cells.

Cell adhesion assay. Specific cell adhesion to recombinant VCAM-1 was performed as previously described by Klein et al (29). Briefly, $0.75 \mu \mathrm{l}$ of $0.5 \mu \mathrm{g} / \mu 1 \mathrm{VCAM}-1$ stock solution was spotted on plastic dishes and immobilized by air-drying. Non-specific binding to plastic dish was prevented by $90 \mathrm{~min}$ pre-incubation with $1 \%$ BSA/PBS. SKOV-3 cells were added for $20 \mathrm{~min}$, partly pre-incubated with $5 \mu \mathrm{g} / \mathrm{ml}$ blocking antibodies for $30 \mathrm{~min}$. Non-adherent cells were removed by gently washing with pre-warmed PBS. Adhesion was evaluated under a Zeiss Axioplan2 microscope. Photographs of representative fields were taken.

Statistical analyses. Fluorescence reader and flow cytometry experiments were conducted at least 3-fold in triplicate. HMCs always derived from three different individuals. The experimental group was analyzed using the t-test, except for antibody blocking experiments (Mann-Whitney rank sum
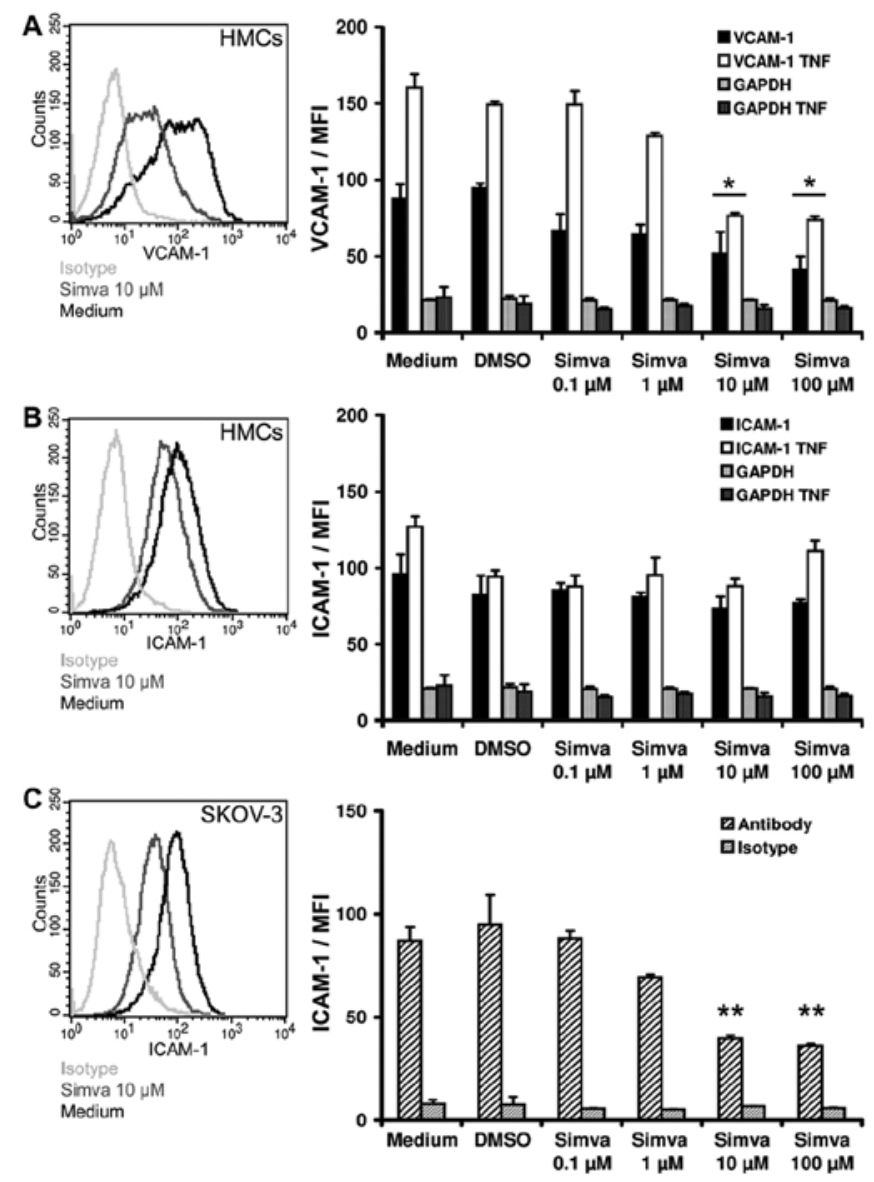

Figure 1. Dose-dependent expression of adhesion molecules on HMCs and SKOV-3 under simvastatin treatment for $48 \mathrm{~h}$. VCAM-1 (A) and ICAM-1 (B) expression after simvastatin (Simva) incubation on HMCs. TNF represents inflammatory conditions in contrast to unstimulated HMCs. Isotype controls did not exceed MFI of 5 for GAPDH or MFI of 10 for ICAM-1/VCAM-1 staining. (C), ICAM-1 expression on SKOV-3 cells. The graphs represent one out of three triplicate stainings. Significance refers to medium and DMSO controls. The data represent means \pm standard deviation.

test). Findings were considered statistically significant at either ${ }^{*} \mathrm{P} \leq 0.05,{ }^{*} \mathrm{P} \leq 0.01$ or ${ }^{* *} \mathrm{P} \leq 0.001$.

\section{Results}

Simvastatin reduces expression of VCAM-1 on HMCs and ICAM-1 on SKOV-3 cells. Maximum down-regulation of VCAM-1 expression on HMCs was observed with $10 \mu \mathrm{M}$ simvastatin after $48 \mathrm{~h}$ (Fig. 1A). Overall, in unstimulated conditions the reduction averaged $41.17 \pm 16.7$ and $34.11 \pm 11.42 \%$ after TNF pre-incubation, compared to DMSO controls. The effect was significant for 10 and $100 \mu \mathrm{M}(\mathrm{P} \leq 0.01)$. The total expression of VCAM-1 was increased by TNF administration. Considering individual basic VCAM-1 levels, the overall mean increase was $27.96 \pm 19.12 \%$. In opposite to VCAM-1, ICAM-1 surface expression on HMCs was not significantly down-regulated by simvastatin (Fig. 1B).

Simvastatin did not reduce GAPDH levels, excluding general inhibition of the cell metabolism. PI staining was performed to prove that simvastatin did not affect cell survival considerably (data not shown). Interestingly, a slight increase of $\sim 3 \%$ in survival of HMCs was observed with simvastatin $10 \mu \mathrm{M}$ versus 

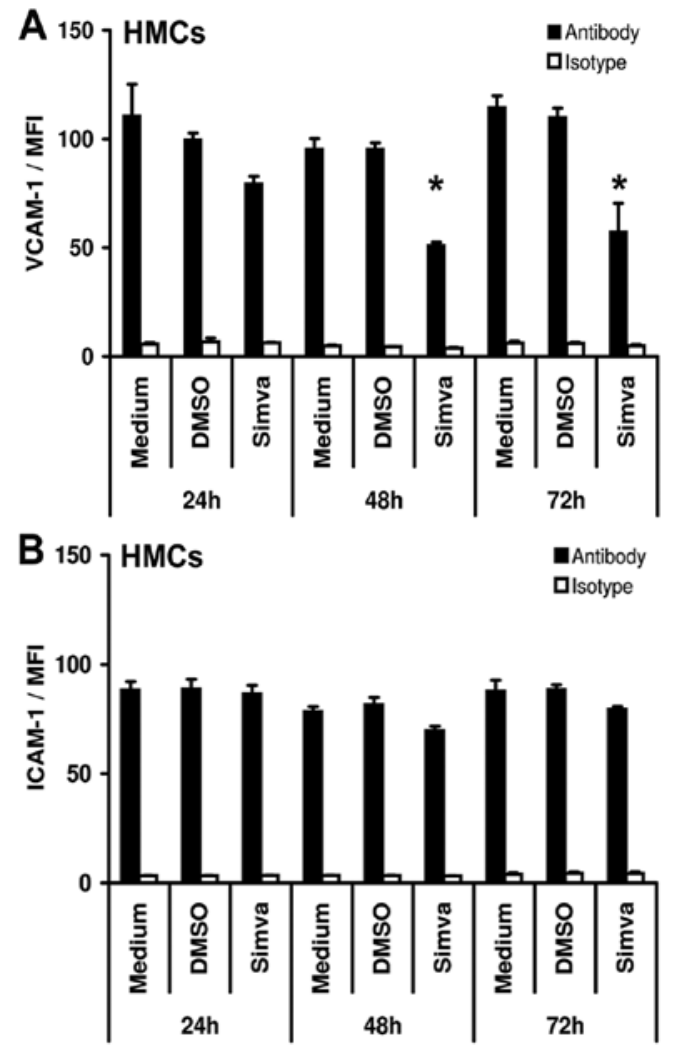

Figure 2. Time-dependent VCAM-1 and ICAM-1 expression on HMCs under simvastatin treatment. All tests were performed after pre-incubation with TNF. VCAM-1 (A) and ICAM-1 (B) expression after $10 \mu \mathrm{M}$ simvastatin (Simva) treatment for 24,48 and $72 \mathrm{~h}$. Significance refers to the corresponding medium and DMSO controls. The data represent one triplicate staining; means are demonstrated \pm standard deviation.

DMSO control to a similar extent after TNF incubation. An adverse effect was detected for the tumor cells. SKOV-3 survival was reduced by $10 \mu \mathrm{M}$ simvastatin by $11.49 \pm 1.44 \%$ in average compared to controls. Similar results were achieved for OvCar-29, FraWü and OAW42 cells.

The ICAM-1 level on SKOV-3 cells was significantly reduced under simvastatin treatment $(\mathrm{P} \leq 0.001)$, (Fig. 1C). The maximum decrease was achieved with $10 \mu \mathrm{M}$ simvastatin after $48 \mathrm{~h}$. ICAM-1 expression was lowered to $\sim 60 \%$. SKOV-3 cells only express minimal levels of VCAM-1, which did not underlie any significant changes during simvastatin treatment (data not shown).

Simvastatin reduces adhesion molecule expression for at least $48 \mathrm{~h}$. Kinetic analyses were carried out at $10 \mu \mathrm{M}$ as simvastatin achieved its maximum effect at this concentration (Fig. 1). The reduction of VCAM-1 expression started about $24 \mathrm{~h}$ after incubation, became significant after $48 \mathrm{~h}$ and lasted up to $72 \mathrm{~h}(\mathrm{P} \leq 0.01)$ (Fig. 2A). No effects were detectable before $24 \mathrm{~h}$. The same was true for ICAM-1 down-regulation on SKOV-3 cells (data not shown). By contrast, a significant decrease in ICAM-1 expression on HMCs (Fig. 2B) was not detected over time. Evaluation was not carried out at longer culture periods to exclude cellular exhaustion due to omission of medium exchanges.

VCAM-1, $\alpha_{4}$ and $\beta_{1}$ integrin chain blockade reduces SKOV-3 adhesion to HMCs. As confirmed for VCAM-1 and ICAM-1
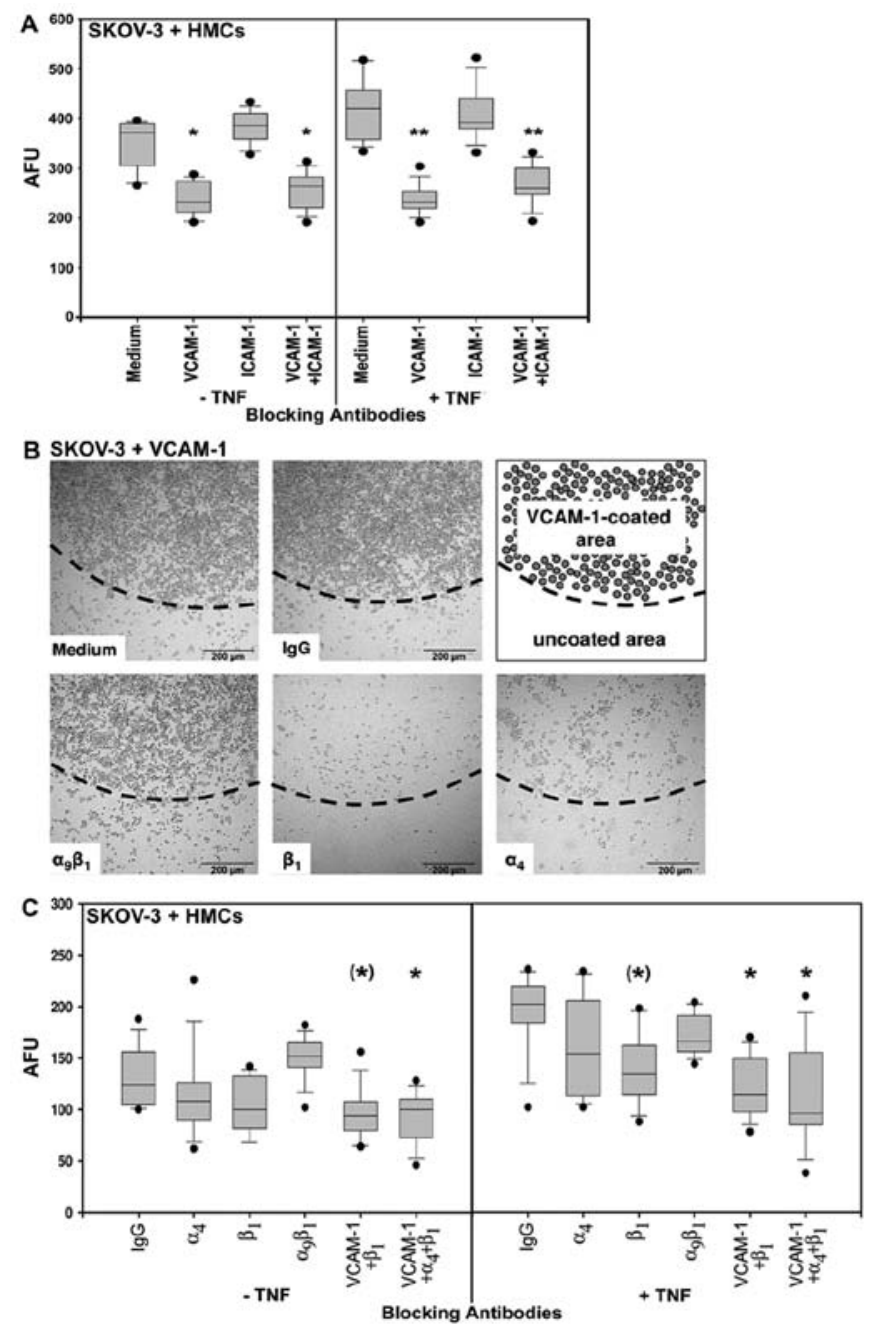

Figure 3. The role of VCAM-1 and its ligands in SKOV-3 adhesion. (A), Adherence of SKOV-3 to HMCs for $48 \mathrm{~h}$ in the presence of ICAM-1 and/or VCAM-1 blocking antibodies. Significance refers to medium controls. All box plots represent the median of three experiments performed in triplicate. - TNF, unstimulated conditions; + TNF, inflammatory conditions. (B), Specific binding of SKOV-3 cells to recombinant VCAM-1 and its reduction by inhibitory antibodies against the integrin $\beta_{1}, \alpha_{4}$ and $\alpha_{9} \beta_{1}$ chains. Photographs of representative fields were taken (magnification x 50). (C), Co-culture of SKOV-3 and HMCs (48 h) with function blocking antibodies directed against the indicated adhesion receptors or IgG control. Significance refers to IgG controls.

on HMCs in this study (Fig. 1) the expression of adhesion molecules is up-regulated by inflammatory stimuli such as TNF $(22,30)$. The effect of inhibitory antibodies and simvastatin (see below) was therefore examined under unstimulated conditions in comparison with TNF pre-incubation of HMCs. Under unstimulated conditions, antibody-mediated VCAM-1 blocking reduced SKOV-3 adhesion to HMCs by $30.42 \pm 12.40 \%$, which was even about $10 \%$ higher under inflammatory conditions (Fig. 3A). The decrease in adherence in comparison with the control medium was significant $(\mathrm{P} \leq 0.01 / 0.001)$. ICAM-1 blocking did not show any effect on SKOV-3 alone, nor did it have any additive effect in combination with VCAM-1 blocking. TNF pre-incubation of HMCs resulted in $15.76 \pm 11.04 \%$ increase of adherent SKOV-3 cells in medium controls.

$\alpha_{4} \beta_{1}$ and $\alpha_{9} \beta_{1}$ integrin are ligands of VCAM-1 $(17,18)$. Adhesion to recombinant VCAM-1 was analyzed to prove that $\beta_{1}, \alpha_{4}$ and 

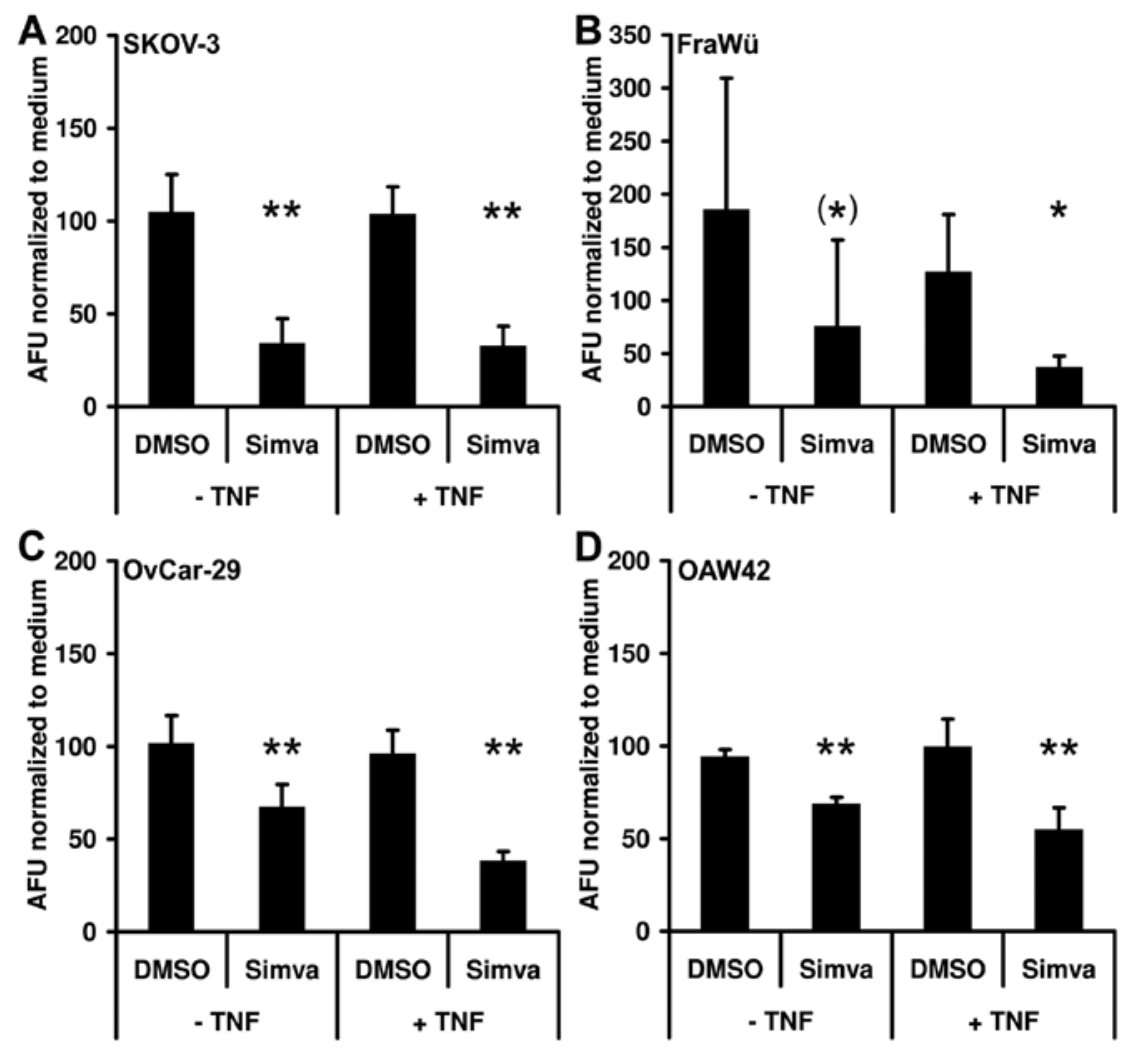

Figure 4. Fluorescence reader analysis of the adhesion of SKOV-3 (A), FraWü (B), OvCar-29 (C) and OAW42 (D) to HMCs. The effect of $10 \mu \mathrm{M}$ simvastatin (Simva) after tumor cell incubation for $48 \mathrm{~h}$ is demonstrated normalized to medium. - TNF, unstimulated conditions; + TNF, inflammatory conditions. The data represent means \pm standard deviation. Significance refers to DMSO controls.

$\alpha_{9}$ integrin chains are required for VCAM-1 mediated adhesion of SKOV-3 cells (Fig. 3B). The light microscopy photographs clearly demonstrate that blocking of $\beta_{1}$ and $\alpha_{4}$ integrin chains resulted in a dramatic inhibition of cell adhesion compared to medium and IgG controls. By contrast blocking of $\alpha_{9} \beta_{1}$ integrin showed almost no effect.

Furthermore, inhibitory antibodies against the integrin $\alpha_{4}$-chain, the $\beta_{1}$-chain and the $\alpha_{9} \beta_{1}$ integrin were also tested to prevent SKOV-3 adhesion to HMCs (Fig. 3C). Blocking of $\alpha_{4}$ and $\beta_{1}$ integrin chains resulted in a decrease in adhesion after $48 \mathrm{~h}$ incubation of approximately one fifth in each case under unstimulated and inflammatory conditions; however these effects were only significant for $\beta_{1}$ integrin chain inhibition after TNF pre-incubation $(\mathrm{P} \leq 0.05)$. The effect was raised to one third and higher by combining $\beta_{1}$ and $\alpha_{4}$ integrin chain inhibition with antibody blockade of VCAM-1 (P $\leq 0.01)$. By contrast, $\alpha_{9} \beta_{1}$ integrin blocking did not decrease adhesion of SKOV-3 cells to HMCs.

Simvastatin reduces ovarian cancer cell adherence to HMCs. After simvastatin incubation, SKOV-3 adhesion was significantly reduced to approximately one third in comparison with DMSO controls in unstimulated conditions and after TNF stimulation $(\mathrm{P} \leq 0.001)$ (Fig. 4A). Simvastatin also reduced adhesion of the ovarian cancer cell lines FraWü (Fig. 4B), OvCar-29 (Fig. 4C) and OAW42 (Fig. 4D). FraWü was the only cell line which showed a considerable increase of adhesion under DMSO compared to medium. Nevertheless, simvastatin lowered FraWü adhesion to HMCs to $<50 \%$ under both condi- tions. OvCar-29 cell adhesion was reduced by $35 \%$ under unstimulated conditions and the effect was almost doubled to $70 \%$ reduction after TNF pre-incubation $(\mathrm{P} \leq 0.001)$. With OAW42 cells the decrease of adhesion was $~ 27 \%$ (unstimulated) and 45\% (inflammatory conditions).

Simvastatin reduces $\beta_{1}$ integrin chain expression on ovarian cancer cells and HMCs. Inhibition of the $\beta_{1}$ integrin chain was the most effective one in reducing SKOV-3 adhesion to its ligand VCAM-1 and to HMCs. Therefore $\beta_{1}$ integrin chain expression on ovarian cancer cells was analyzed under simvastatin treatment (Fig. 5A). Simvastatin reduced the $\beta_{1}$ integrin chain expression significantly on all ovarian cancer cells $(\mathrm{P} \leq 0.01)$. It reached its maximum at $10 \mu \mathrm{M}$ after $48-\mathrm{h}$ incubation. The highest effect was achieved with OvCar-29 cells. $\beta_{1}$ integrin chain expression was reduced to $<50 \%$ compared to controls $(\mathrm{P} \leq 0.001)$. Further $10 \mu \mathrm{M}$ simvastatin also reduced $\beta_{1}$ integrin chain expression on HMCs about one third under inflammatory and unstimulated condition (Fig. 5B); however this effect was only significant after TNF pre-incubation $(\mathrm{P} \leq 0.05)$.

Simvastatin does not influence ICAM-1 expression on HT29 cells. Simvastatin did not significantly reduce ICAM-1 expression on HT29 cells at any dosages after $48 \mathrm{~h}$ (Fig. 6A) or during kinetic analyses (data not shown). VCAM-1 was not expressed on HT29 (data not shown). PI-staining proved that survival of HT29 cells was slightly decreased by $7.16 \pm 6.94 \%$ under simvastatin treatment compared to controls (data not shown). 

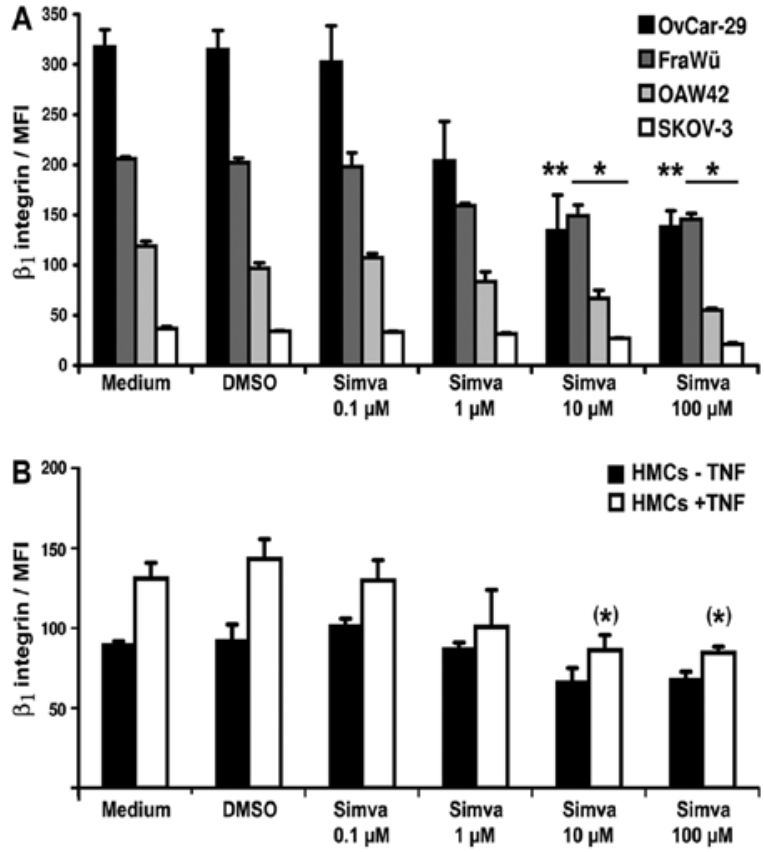

Figure 5. Dose-dependent expression of $\beta_{1}$ integrin chain on ovarian cancer cells (A) and HMCs (B) under simvastatin treatment for $48 \mathrm{~h}$. Isotype controls did not exceed MFI of 13 in ovarian cancer cells and MFI of 10 in HMCs. - TNF, unstimulated conditions; + TNF, inflammatory conditions. Significance refers to the corresponding medium and DMSO controls. The data represent one triplicate staining; the means are demonstrated \pm standard deviation.
HT29 adhesion to HMCs is reduced by ICAM-1 inhibition and simvastatin treatment. HT29 cell adherence to HMCs was only decreased by ICAM-1 blocking (Fig. 6B). In unstimulated conditions, ICAM-1 inhibition reduced HT29 adhesion by $26.26 \pm 4.22 \%$, which was elevated about $10 \%$ after TNF preincubation. Comparing medium controls, under inflammatory conditions total adhesion of HT29 was increased about one third. VCAM-1 inhibition did not show an effect alone nor in combination with ICAM-1.

Simvastatin only reduced adhesion of HT29 cells to HMCs to $\sim 50 \%$ under unstimulated conditions ( $\mathrm{P} \leq 0.001)$, (Fig. 6C). By contrast, after TNF pre-incubation simvastatin did not decrease HT29 adhesion compared to DMSO.

\section{Discussion}

The data reported here uncover simvastatin as a promising agent for preventing cancer cell adhesion to HMCs. For this purpose the reduction of VCAM-1 and $\alpha_{4} \beta_{1}$ integrin expression by simvastatin has to be considered as major molecular mechanism. This may provide a new possibility to prevent intra-abdominal tumor cell adhesion and peritoneal carcinomatosis.

Microscopic intra-peritoneal tumor lesions often elude surgical treatment (13). They are regarded as one cause of peritoneal carcinomatosis (12-14). Cancer cell dissociation
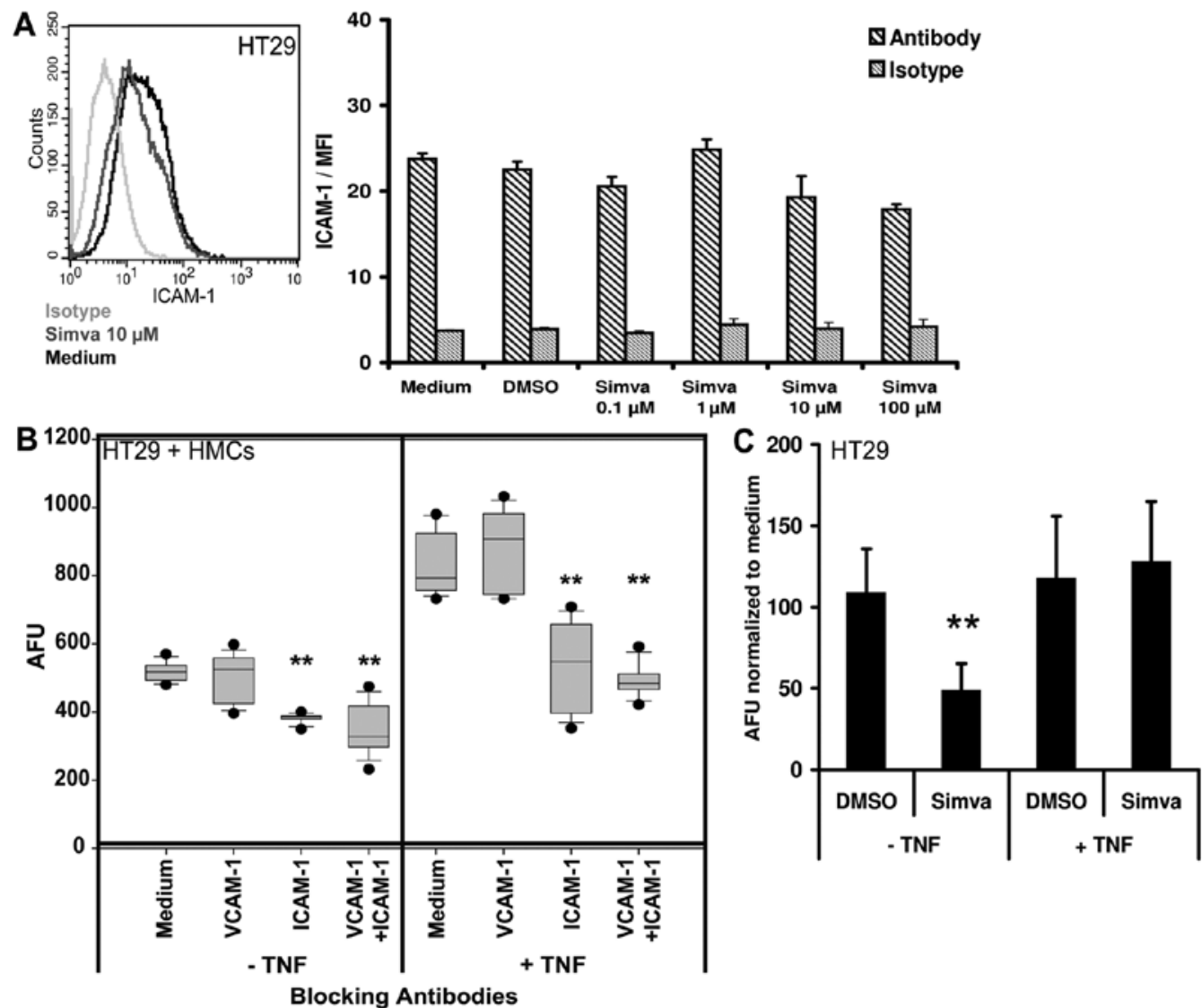

Figure 6. ICAM-1 expression and adhesion of HT29 under simvastatin treatment. (A), Dose-dependent surface expression of ICAM-1 on HT29 cells under simvastatin treatment for $48 \mathrm{~h}$. The data represent one triplicate staining; the means are shown \pm standard deviation. (B), Influence of VCAM-1 and/or ICAM-1 blocking antibodies on HT29 adhesion to HMCs. Significance refers to medium controls. (C), Effect of $10 \mu \mathrm{M}$ simvastatin (Simva) on HT29 adhesion to HMCs. - TNF, unstimulated conditions; + TNF, inflammatory conditions. Adherence was always measured after 48-h incubation. The data represent the medians or means \pm standard deviation. 
from the primary tumor or their exfoliation during surgery are the first steps of peritoneal carcinomatosis $(12,13,31)$. Besides intraoperative cytotoxic strategies, a promising method to avoid intra-peritoneal inoculation seems to be the inhibition of cancer cell adhesion to HMCs $(17,19)$.

The adhesion molecules VCAM-1, ICAM-1 and members of the $\beta_{1}$ integrin subfamily appear to be promising targets to reduce intra-abdominal metastasis. Simvastatin reduced the level of VCAM-1 expression on HMCs and $\beta_{1}$ integrin chain levels on ovarian cancer cells and HMCs significantly. VCAM-1, $\alpha_{4}$ and $\beta_{1}$ integrin chains blocking decreased adherence of SKOV-3 cells to HMCs. Adhesion of SKOV-3 to HMCs was also substantially reduced by simvastatin to one third. This also accounts to similar extent for all further tested ovarian cancer cell lines. These observations provide proof-of-principle evidence that simvastatin decreases SKOV-3 adhesion to HMCs via VCAM-1 down-regulation on HMCs and reduced expression of its ligand $\alpha_{4} \beta_{1}$ integrin on the ovarian cancer cells tested. Further ICAM-1 expression on SKOV-3 cells also declined during simvastatin treatment; however ICAM-1 blockade could not prevent SKOV-3 adhesion to HMCs. Therefore, it is unlikely that it is involved in decreased SKOV-3 adhesion to HMCs.

Interestingly, simvastatin reduced the adhesion of HT29 colorectal tumor cells to HMCs only under unstimulated conditions but not after TNF pre-incubation. Furthermore, HT29 adhesion to HMCs was reduced by ICAM-1 blocking. ICAM-1 expression on HMCs has been described to play a central role in the adhesion process of HT29 (19,32). LFA (lymphocyte function-associated antigen)-1 is a member of the $\beta_{2}$ integrin subfamily and a ligand of ICAM-1. It is expressed on the surface of HT29 cells $(32,33)$. Despite this, simvastatin had no effect on ICAM-1 expression, either on HMCs or on HT29 cells. The influence of simvastatin on HT29 adhesion to HMCs thus appears to be independent of ICAM-1 expression.

Adhesion molecule blocking antibodies have successfully been used to decrease tumor cell-host cell interactions and consequently reduced tumor metastasis in vivo $(17,19,20)$. Therefore, they are regarded as promising targets for therapeutic intervention $(17,19,20)$. Nevertheless, intercellular adhesion requires different adhesion molecules at the same time $(17,33,34)$. In contrast to inhibitory antibodies, which only target one defined adhesion molecule, simvastatin lowered different adhesion molecules on ovarian cancer cells and HMCs contemporaneously. This explains that simvastatin could reduce SKOV-3 adhesion to HMCs to one third although antibody blocking of VCAM-1 and/or $\beta_{1}$ integrin only resulted in a $40 \%$ maximum decrease. Nevertheless simvastatin does not generally lower surface molecule expression but rather seems to reduce selective molecules. ICAM-1 expression on HMCs stayed unchanged; however, VCAM-1 was clearly down-regulated.

Certain statins, including simvastatin, induce cell death in ovarian cancer cells in vitro (35-37). Therefore, PI staining was performed to exclude that the decreased numbers of adherent tumor cells by simvastatin were only due to cell death induction. The survival of ovarian cancer cells and HT29 was indeed slightly lowered. Nevertheless about $10 \%$ reduced vitality could not account for $30-70 \%$ less tumor cell adhesion alone. Consequently the reduction of tumor cell adhesion to HMCs by simvastatin seemed to be primarily due to a decreased surface expression of adhesion molecules.
Physical stress, for example by surgery, or chemical injury to the peritoneum, provokes a sterile local inflammation, which is a privileged site of tumor recurrence $(10,16,38,39)$. The up-regulation of adhesion molecules on HMCs is regarded as causal mechanism behind this observance $(30,40)$. Simvastatin decreased ovarian cancer cell adherence to HMCs under unstimulated and TNF pre-incubated conditions. This finding underlines that it may be a promising substance for intraabdominal application after surgery to prevent the adhesion of tumor cells to HMCs. Further, simvastatin did not impinge upon the vitality or the metabolism of HMCs after 48-72-h treatment. Equal GAPDH levels in controls and under simvastatin proved that down-regulation of adhesion molecules on HMCs was not due to general inhibition of cell metabolism. Therefore it seems improbable that simvastatin would cause additional inflammatory stress to the HMC monolayer in vivo. In addition, the long-lasting effect of simvastatin of at least $48 \mathrm{~h}$ emphasizes potential benefits in peri-operative settings.

The use of statins for cardiovascular indications and a coeval reduction of cancer incidence has been reported $(22,23)$. In a retrospective study, statins improved overall survival in patients with epithelial ovarian cancer (41). By contrast, a recent retrospective cohort study failed to show a correlation between statin use and reduced ovarian cancer incidence (42). Results are also inconsistent for colorectal cancer. In a case-control study including 1953 cancer patients and 2015 controls, the use of statins was associated with $47 \%$ relative reduction in the risk of colorectal cancer (43). Nevertheless, a systematic review of 42 randomized trials states that statins have protective effects on only certain cancers like stomach, liver and lymphoma but not on colorectal cancer (44). To date little is known about the molecular mechanisms of simvastatin, which are responsible for its anti-cancer effects. The current findings suggest that down-regulation of adhesion molecules, especially of VCAM-1 and $\beta_{1}$ integrin chain may be involved, however this still has to be proven for systemic application. As simvastatin decreased ovarian cancer and HT29 tumor cell adhesion to HMCs significantly, besides systemic use, direct local application of simvastatin may be considered as a promising option to prevent intra-peritoneal cancer cell adhesion.

Overall, as an example of adhesion molecule down-regulating drugs in general, simvastatin may be a promising agent for preventing peritoneal carcinomatosis. Since we found the effect on several ovarian cancer cell lines, simvastatin treatment might warrant evaluation in patients with ovarian cancer, in particular in VCAM-1 and $\alpha_{4} \beta_{1}$ integrin-dependent tumor metastasis.

\section{Acknowledgments}

We thank Tobias Feuchtinger and Amelie Halder (Department of Pediatrics, University of Tübingen, Germany) for technical support with fluorescence reader analysis and Bettina HasslingerLöffler and Michaela Brück (Institute of Microbiology, University of Münster, Germany) for sharing their expertise in working with HMCs. Further grant support was provided by fortüne-funding (1793-0-1), Medical Faculty, University of Tübingen, Germany (B. Wagner) and by the Deutsche Forschungsgemeinschaft grant SFB 685/Z3, Department of Immunology, University of Tübingen, Germany (B. Wagner, S. Löb). 


\section{References}

1. Sugarbaker PH: A perspective on clinical research strategies in carcinoma of the large bowel. World J Surg 15: 609-616, 1991.

2. Bell DA, Longacre TA, Prat J, et al: Serous borderline (low malignant potential, atypical proliferative) ovarian tumors: workshop perspectives. Hum Pathol 35: 934-948, 2004.

3. Agarwal R and Kaye SB: Ovarian cancer: strategies for overcoming resistance to chemotherapy. Nat Rev Cancer 3: 502-516, 2003.

4. Daly MB and Ozols RF: Symptoms of ovarian cancer - where to set the bar? JAMA 291: 2755-2756, 2004.

5. Ozols RF: Treatment goals in ovarian cancer. Int J Gynecol Cancer 15: 3-11, 2005.

6. Koppe MJ, Boerman OC, Oyen WJ and Bleichrodt RP: Peritoneal carcinomatosis of colorectal origin: incidence and current treatment strategies. Ann Surg 243: 212-222, 2006.

7. Esquivel J, Sticca R, Sugarbaker P, et al: Cytoreductive surgery and hyperthermic intraperitoneal chemotherapy in the management of peritoneal surface malignancies of colonic origin: a consensus statement. Society of Surgical Oncology. Ann Surg Oncol 14: 128-133, 2007

8. Verwaal VJ, Boot H, Aleman BM, van Tinteren $\mathrm{H}$ and Zoetmulder FA: Recurrences after peritoneal carcinomatosis of colorectal origin treated by cytoreduction and hyperthermic intraperitoneal chemotherapy: location, treatment, and outcome. Ann Surg Oncol 11: 375-379, 2004.

9. Kenny HA, Krausz T, Yamada SD and Lengyel E: Use of a novel 3D culture model to elucidate the role of mesothelial cells, fibroblasts and extra-cellular matrices on adhesion and invasion of ovarian cancer cells to the omentum. Int J Cancer 121: 1463-1472, 2007.

10. Oosterling SJ, van der Bij GJ, Bogels M, et al: Anti-beta1 integrin antibody reduces surgery-induced adhesion of colon carcinoma cells to traumatized peritoneal surfaces. Ann Surg 247: 85-94, 2008.

11. Busch OR, Hop WC, Hoynck van Papendrecht MA, Marquet RL and Jeekel J: Blood transfusions and prognosis in colorectal cancer. N Engl J Med 328: 1372-1376, 1993.

12. Kanellos I, Demetriades H, Zintzaras E, Mandrali A, Mantzoros I and Betsis D: Incidence and prognostic value of positive peritoneal cytology in colorectal cancer. Dis Colon Rectum 46: 535-539, 2003.

13. Sugarbaker PH: Intraperitoneal chemotherapy for treatment and prevention of peritoneal carcinomatosis and sarcomatosis. Dis Colon Rectum 37: 115-122, 1994.

14. Yamamoto S, Akasu T, Fujita S and Moriya Y: Long-term prognostic value of conventional peritoneal cytology after curative resection for colorectal carcinoma. Jpn J Clin Oncol 33: 33-37, 2003.

15. Jonjić N, Peri G, Bernasconi S, et al: Expression of adhesion molecules and chemotactic cytokines in cultured human mesothelial cells. J Exp Med 176: 1165-1174, 1992.

16. ten Raa RS, van Grevenstein HM, ten Kate M, et al: The influence of reactive oxygen species on the adhesion of pancreatic carcinoma cells to the peritoneum. Cell Adh Migr 1: 77-83, 2007.

17. Slack-Davis JK, Atkins KA, Harrer C, Hershey ED and Conaway M: Vascular cell adhesion molecule-1 is a regulator of ovarian cancer peritoneal metastasis. Cancer Res 69: 1469-1476, 2009.

18. Lessan K, Aguiar DJ, Oegema T, Siebenson L and Skubitz AP CD44 and beta1 integrin mediate ovarian carcinoma cell adhesion to peritoneal mesothelial cells. Am J Pathol 154: 1525-1537, 1999.

19. Alkhamesi NA, Ziprin P, Pfistermuller K, Peck DH and Darzi AW: ICAM-1 mediated peritoneal carcinomatosis, a target for therapeutic intervention. Clin Exp Metastasis 22: 449-459, 2005.

20. Dianzani C, Brucato L, Gallicchio M, Rosa AC, Collino M and Fantozzi R: Celecoxib modulates adhesion of HT29 colon cancer cells to vascular endothelial cells by inhibiting ICAM-1 and VCAM-1 expression. Br J Pharmacol 153: 1153-1161, 2008.

21. Moorman PG and Hamilton RJ: Statins and cancer risk: what do we know and where do we go from here? Epidemiology 18: 194-196, 2007.

22. Graaf MR, Beiderbeck AB, Egberts AC, Richel DJ and Guchelaar HJ: The risk of cancer in users of statins. J Clin Oncol 22: 2388-2394, 2004.

23. Sassano A and Platanias LC: Statins in tumor suppression. Cancer Lett 260: 11-19, 2008
24. Landsberger M, Wolff B, Jantzen F, et al: Cerivastatin reduces cytokine-induced surface expression of ICAM-1 via increased shedding in human endothelial cells. Atherosclerosis 190: 43-52, 2007.

25. Zapolska-Downar D, Siennicka A, Kaczmarczyk M, Kolodziej B and Naruszewicz M: Simvastatin modulates TNFalpha-induced adhesion molecules expression in human endothelial cells. Life Sci 75: 1287-1302, 2004.

26. Takeda I, Maruya S, Shirasaki T, et al: Simvastatin inactivates betal-integrin and extracellular signal-related kinase signaling and inhibits cell proliferation in head and neck squamous cell carcinoma cells. Cancer Sci 98: 890-899, 2007.

27. Stylianou E, Jenner LA, Davies M, Coles GA and Williams JD: Isolation, culture and characterization of human peritoneal mesothelial cells. Kidney Int 37: 1563-1570, 1990.

28. Kayser S, Watermann I, Rentzsch C, Weinschenk T, Wallwiener D and Gückel B: Tumor-associated antigen profiling in breast and ovarian cancer: mRNA, protein or T cell recognition? J Cancer Res Clin Oncol 129: 397-409, 2003.

29. Klein G, Muller CA, Tillet E, Chu ML and Timpl R: Collagen type VI in the human bone marrow microenvironment: a strong cytoadhesive component. Blood 86: 1740-1748, 1995.

30. Van Grevenstein WM, Hofland LJ, Jeekel J and van Eijck CH: The expression of adhesion molecules and the influence of inflammatory cytokines on the adhesion of human pancreatic carcinoma cells to mesothelial monolayers. Pancreas 32: 396-402, 2006.

31. Tan DS, Agarwal R and Kaye SB: Mechanisms of transcoelomic metastasis in ovarian cancer. Lancet Oncol 7: 925-934, 2006.

32. Van Grevenstein WM, Hofland LJ, van Rossen ME, van Koetsveld PM, Jeekel J and van Eijck $\mathrm{CH}$ : Inflammatory cytokines stimulate the adhesion of colon carcinoma cells to mesothelial monolayers. Dig Dis Sci 52: 2775-2783, 2007.

33. ten Kate M, Hofland LJ, van Grevenstein WM, van Koetsveld PV, Jeekel $\mathrm{J}$ and van Eijck $\mathrm{CH}$ : Influence of proinflammatory cytokines on the adhesion of human colon carcinoma cells to lung microvascular endothelium. Int J Cancer 112: 943-950, 2004

34. Wang E, Ngalame Y, Panelli MC, et al: Peritoneal and subperitoneal stroma may facilitate regional spread of ovarian cancer. Clin Cancer Res 11: 113-122, 2005.

35. Han Z and Wyche JH: Lovastatin induces apoptosis in a metastatic ovarian tumour cell line. Cell Death Differ 3: 223-228, 1996.

36. Liu H, Liang SL, Kumar S, Weyman CM, Liu W and Zhou A: Statins induce apoptosis in ovarian cancer cells through activation of JNK and enhancement of Bim expression. Cancer Chemother Pharmacol 63: 997-1005, 2009.

37. Kato S, Smalley S, Sadarangani A, et al: Lipophilic but not hydrophilic statins selectively induce cell death in gynecological cancers expressing high levels of HMGCoA reductase. J Cell Mol Med 14: $1180-1193,2010$

38. Van Grevenstein WM, Aalbers AG, ten Raa S, et al: Surgeryderived reactive oxygen species produced by polymorphonuclear leukocytes promote tumor recurrence: studies in an in vitro model. J Surg Res 140: 115-120, 2007.

39. Königsrainer I, Zieker D, Beckert S, et al: Local peritonectomy highly attracts free floating intraperitoneal colorectal tumour cells in a rat model. Cell Physiol Biochem 23: 371-378, 2009.

40. Ziprin P, Ridgway PF, Pfistermuller KL, Peck DH and Darzi AW: ICAM-1 mediated tumor-mesothelial cell adhesion is modulated by IL- 6 and TNF-alpha: a potential mechanism by which surgical trauma increases peritoneal metastases. Cell Commun Adhes 10: $141-154,2003$.

41. Elmore RG, Ioffe Y, Scoles DR, Karlan BY and Li AJ: Impact of statin therapy on survival in epithelial ovarian cancer. Gynecol Oncol 111: 102-105, 2008.

42. Yu O, Boudreau DM, Buist DS and Miglioretti DL: Statin use and female reproductive organ cancer risk in a large populationbased setting. Cancer Causes Control 20: 609-616, 2009.

43. Poynter JN, Gruber SB, Higgins PD, et al: Statins and the risk of colorectal cancer. N Engl J Med 352: 2184-2192, 2005.

44. Kuoppala J, Lamminpaa A and Pukkala E: Statins and cancer: a systematic review and meta-analysis. Eur J Cancer 44: 2122-2132, 2008. 